Working Paper/Document de travail 2013-7

\title{
A Tractable Monetary Model Under General Preferences
}

by Tsz-Nga Wong 
Bank of Canada Working Paper 2013-7

March 2013

\title{
A Tractable Monetary Model Under General Preferences
}

\author{
by \\ Tsz-Nga Wong \\ Funds Management and Banking Department \\ Bank of Canada \\ Ottawa, Ontario, Canada K1A 0G9 \\ rwong@bankofcanada.ca
}

Bank of Canada working papers are theoretical or empirical works-in-progress on subjects in economics and finance. The views expressed in this paper are those of the author.

No responsibility for them should be attributed to the Bank of Canada. 


\section{Acknowledgements}

I would like to thank helpful comments from Jonathan Chiu, Ricardo Lagos, Miguel Molico, Guillaume Rocheteau, Enchuan Shao, Randy Wright and Steve Williamson. I am responsible for all errors and omissions. 


\begin{abstract}
Consider the monetary model of Lagos and Wright (JPE 2005) but with general preferences and general production. I show that preferences satisfying $U_{X X} U_{H H}-\left(U_{X H}\right)^{2}=$ 0 is a sufficient condition for the existence and uniqueness of monetary equilibrium with degenerate money distribution. I solve for the entire class of exact solutions to the above non-linear second order partial differential equation. This class of preferences includes ones with constant return to scale, for example, constant elasticity of substitution (CES), and ones used in many other macroeconomics literatures. I also analyze the welfare implication of monetary policy in this economy.
\end{abstract}

JEL classification: E40, D83

Bank classification: Economic models

\title{
Résumé
}

En se fondant sur le modèle monétaire de Lagos et Wright (JPE, 2005), mais en introduisant des préférences générales et une production générale, l'auteur montre que les préférences qui satisfont l'équation différentielle partielle et non linéaire du second ordre $U_{X X} U_{H H}-\left(U_{X H}\right)^{2}=0$ forment une condition suffisante de l'existence et de l'unicité d'un équilibre monétaire avec distribution dégénérée de la monnaie. Il résout l'équation pour une classe entière de solutions exactes. Cette classe de préférences comporte des préférences à rendements d'échelle constants (cas de l'élasticité de substitution constante dite CES) et des préférences comme en énoncent de nombreux travaux de macroéconomie. L’auteur analyse également les incidences de la politique monétaire sur le bien-être des agents de son modèle.

Classification JEL : E40, D83

Classification de la Banque : Modèles économiques 


\section{Introduction}

In recent years, a significant literature on monetary theory has emerged to study how money arises endogenously. One such strand of the literature has used search-theoretic models of monetary exchange to understand the role of fundamental frictions such as spatial, temporal or information frictions, lack of commitment and lack of recordkeeping that make money essential. For example, see Kiyotaki and Wright (1989), Trejos and Wright (1995) and Shi (1995) for the pioneers of this literature. However, a challenge in the literature is to keep track of the endogenous distribution of money, which makes policy analysis difficult without restricting the divisibility of money and goods, or relying on sophisticated computation methods, see Molico (2006). ${ }^{1}$ It was not until the seminal work by Lagos and Wright (2005, hereby LW) that researchers can work with an environment which allows divisible money and divisible goods, but at the same time keeps the distribution of money balance degenerate. The key trick in LW is to assume quasi-linear preferences. ${ }^{2}$ However, quasi-linear utility is incompatible with many useful utility functions, for example, the one with constant elasticity of substitution between consumption and leisure. In other words, one might worry that modern monetary theories based on LW are not compatible with many other macroeconomic theories based on general preferences, and of limited application. Furthermore, in LW there is no wealth effect on consumption - any wealth effect only reflects on change in labor supply. Also, since utility must be separable in LW, there is no substitution nor complementary effect between consumption and labor through the preferences channel. Such features cast some doubt on the robustness of the result derived, if other preferences are used instead.

This paper shows that quasi-linear utility is sufficient but not necessary for tractability. In particular, this paper identifies a broad class of preferences which features a degenerate money distribution in equilibrium. This class of preferences represents a general solution to a nonlinear second order partial differential equation,

\footnotetext{
${ }^{1}$ For exmaple, Kiyotaki and Wright (1989) assume indivisible money and indivisible goods, and Trejos and Wright (1995) for indivisible money and divisible goods.

${ }^{2}$ Also see Shi (1997) for a "big household" approach. Chiu and Molico (2010) performs numerical analysis when the quasi-linear assumption is violated.
} 
$U_{X X} U_{H H}-\left(U_{X H}\right)^{2}=0$, whose particular solutions include utility with constant elasticity of substitution, non-separable CARA, quasi-linear, and many other - preferences which are commonly adopted in many other literatures like finance, endogenous growth, and endogenous fertility. This finding suggests that previous results based on quasi-linear utility are more robust than one might have thought- LW is a member of a wider class of economies rather than just a singularity. As an illustration, this paper also checks the qualitative implications of monetary policy in a LW economy under general preferences.

There are also previous works in another direction: provide particular examples where the economy in some equilibria looks like LW, instead of exploring the most general environment sufficient to establish degenerate equilibrium. Both directions are helpful to expand the applicability of the search-theoretic model of money. Rocheteau et al (2008) consider an economy with indivisible goods where, under the sunspot equilibria, a type of correlated equilibrium, agents act as if they have quasi-linear utility function. Similar properties are also shown by Faig (2008), which depends on the lottery of agents' money balances. A useful trick of these works is to exploit the non-concavity of the model: if there is a randomization device and agents can coordinate on it, then there is a region such that the value function is linear, hence it looks like quasi-linear preferences.

\section{The Model}

Consider the similar environment as in the LW model. Time is discrete and infinite, indexed by $t=0,1 \ldots$ There is a $[0,1]$ continuum of agents who live forever with discount factor $\beta \in(0,1)$. In each period, two markets, DM and CM, convene subsequently. Trading in the DM is decentralized and bilateral (agents are paired with probability $\alpha) .{ }^{3}$ Some agents (sellers) can produce a divisible and perishable goods but do not want to consume, while others (buyers) want to consume but cannot produce, hence trading emerges. Agents can only observe the actions and outcomes

\footnotetext{
${ }^{3}$ The main result still holds when DM in this economy is modified to incorporate competitive search equibrium or competitive equilibrium as in Rocheteau and Wright (2005).
} 
of their trades, and are anonymous. ${ }^{4}$ There is no commitment nor enforcement. Trading in the CM is centralized. All agents receive government money transfer, work, consume, rebalance their money at some market-clearing prices. Money is divisible, intrinsically worthless and non-perishable, with initial supply $M_{0}$ for each agents and subsequent supply controlled by the government. This setting is useful to capture the endogenous rise of money as the medium of exchange.

Instead of assuming quasi-linear preferences as in LW, we formulate the instantaneous utility by the following general utility function:

$$
u(x, h)+U(X, H)
$$

where $X$ and $H(x$ and $h)$ are consumption and labor during the CM (DM). We maintain the following standard assumptions that the economy is "smooth", and there exists some trade in the DM with positive total surplus (otherwise no trade in the DM is preferable):

Assumption 1 Assume $u$ and $U$ are $C^{n}$ with $n \geq 2$. Also, assume $U_{X}>0, U_{H}<$ $0, U_{X X}<0, U_{H H} \leq 0, u_{x}>0, u_{h}<0, u_{x x}<0, u_{h h} \leq 0$ and $u(U)$ satisfies Inada condition in $x(X)$. Also, assume $u(0,0)=0$, and $\max _{q}\{u(q, 0)+u(0, q)\}>0$.

I begin by describing the value functions, taking as given the terms of trade and the distribution of money. In general, the state variable relevant for an agent in the $\mathrm{DM}$ is a vector of aggregate states $S_{t} \equiv\left(\phi_{t}, w_{t}, G_{t}\left(\phi_{t} m_{t}\right)\right)$, where $\phi_{t}$ is the value of money in the centralized market, $w_{t}$ the wage of labor, and $G_{t}$ the cumulative distribution function of the real money balances $\phi_{t} m_{t}$ held by agents in the DM. The agent takes as given a law of motion $S_{t+1}=\Psi\left(S_{t}\right)$, but it will be determined endogenously in equilibrium.

In the DM, with probability $\alpha \leq 1 / 2$, the agent matches a seller and consumes $x$ goods with utility $u(x, 0)$. By symmetry, there is probability $\alpha$ that the agent matches a buyer and producers $h$ goods with disutility $u(0, h)$. In a match in the

\footnotetext{
${ }^{4}$ Money may not be essential when agents can also observe other non-trading agents' action or can coordinate a global punishment strategy. See Aliprantis et al (2007) and Sanches and Williamson (2010). See Kocherlakota (1998) on the role of recordkeeping in a monetary model.
} 
DM, since the seller's production $h$ must equal the buyer's consumption $x$, their common value is denoted by $q_{t}\left(z, z^{\prime}, S_{t}\right)$, and the real money balances that change hands by $d_{t}\left(z, z^{\prime}, S_{t}\right)$, where in general these depend on the real money holdings of the buyer $z$ and of the seller $z^{\prime}$ as well as the aggregate state $S_{t}$. Unless potential confusion arises, I suppress the dependence on $S_{t}$. At the beginning of DM, given his real money balances $z$, the agent's value function $V_{t}$ is the Bellman equation solving

$$
\begin{aligned}
V_{t}(z)= & \alpha \int\left\{u\left(q_{t}\left(z, z^{\prime}\right), 0\right)+W_{t}\left(z-d_{t}\left(z, z^{\prime}\right)\right)\right\} d G_{t}\left(z^{\prime}\right) \\
& +\alpha \int\left\{u\left(0, q_{t}\left(z^{\prime}, z\right)\right)+W_{t}\left(z+d_{t}\left(z^{\prime}, z\right)\right)\right\} d G_{t}\left(z^{\prime}\right) \\
& +(1-2 \alpha) W_{t}(z) .
\end{aligned}
$$

At the beginning of $\mathrm{CM}$, the agent $i$ 's value function $W_{t}$ is the Bellman equation solving

$$
\begin{aligned}
W_{t}(z) & =\max _{X_{t}^{i}, H_{t}^{i}, z_{t+1}^{i}}\left\{U\left(X_{t}^{i}, H_{t}^{i}\right)+\beta V_{t+1}\left(z_{t+1}^{i}\right)\right\}, \\
\text { s.t. } X_{t}^{i} & =w_{t} H_{t}^{i}+z-\left(1+\pi_{t+1}\right) z_{t+1}^{i}+T_{t}+\Pi_{t},
\end{aligned}
$$

where $\pi_{t+1} \equiv \phi_{t} / \phi_{t+1}-1$ is the inflation rate, $T_{t}$ the real government transfer, $z_{t+1}^{i}$ the real money balances carried into the $\mathrm{DM}$ in $t+1$, and $\Pi_{t}$ the dividends from firms.

Now, for the illustration purpose, consider the generalized Nash bargaining for matches in the DM, which is commonly used in the literature. For a buyer with the real money balances $z$ who matches the seller with the real money balances $z^{\prime}$ in the $\mathrm{DM},\left(q_{t}\left(z, z^{\prime}\right), d_{t}\left(z, z^{\prime}\right)\right)$ solves

$$
\max _{q_{t}, d_{t} \leq z}\left[u\left(q_{t}, 0\right)+W_{t}\left(z-d_{t}\right)-W_{t}(z)\right]^{\eta}\left[u\left(0, q_{t}\right)+W_{t}\left(z^{\prime}+d_{t}\right)-W_{t}\left(z^{\prime}\right)\right]^{1-\eta},
$$

where the buyer's bargaining power is given by $\eta \in(0,1]$.

During the CM, the firm has access to a general production technology $X=F(H)$ 
and hires agents at wage $w_{t}{ }^{5}$ The firm maximizes profit $\Pi_{t}$ :

$$
\Pi_{t} \equiv \max _{H_{t}}\left\{F\left(H_{t}\right)-w_{t} H_{t}\right\}
$$

where $H_{t}=\int_{i \in[0,1]} H_{t}^{i} d i$ is the aggregate labor supply of agents. We maintain the following assumption to ensure Pareto optimum allocation in a centralized market exists and unique, and the Pareto optimum allocation is sufficient large to support some monetary trades in the DM:

Assumption 2 There exists unique $X^{*} \in(0, \infty)$ and $H^{*} \in(0, \infty)$ that maximize $U\left(X^{*}, H^{*}\right)$ subject to $X^{*}=F\left(H^{*}\right)$. Define $\theta^{*}=U_{X}\left(X^{*}, H^{*}\right)$. Also, define $\bar{z} \equiv \max _{X, H}\left\{F\left(H^{*}\right)+F_{H}\left(H^{*}\right)\left(H-H^{*}\right)-X\right\}$ subject to $\theta^{*}=U_{X}(X, H)$. Assume $\bar{z} \geq u\left(q^{*}, 0\right) / \theta^{*}$.

The government injects money $T_{t} / \phi_{t}=M_{t+1}-M_{t}$ as lump sum transfer in the CM. Let $\tau \equiv M_{t+1} / M_{t}-1$ denote the growth rate of money supply. In the equilibrium, money demand equals to the money supply, ie $z_{t} \equiv \phi_{t} M_{t}=\int_{i \in[0,1]} z_{t}^{i} d i$, and the government budget becomes:

$$
T_{t}=\tau z_{t}
$$

which is assumed always satisfied.

Also, for later use, define a function $Z(q)$ as

$$
Z(q) \equiv-u(0, q)+(1-\eta) \frac{u(q, 0)+u(0, q)}{1-\eta\left[1+\frac{u_{x}(q, 0)}{u_{h}(0, q)}\right]}
$$

Here $Z(q)$ will be shown to be the payment to the seller for $q$ goods, adjusting for the bargaining power. The seller extracts all the trade surplus when $\eta=0$ and hence $Z(q)=u(0, q)$; zero trade surplus when $\eta=1$ and $Z(q)=-u(0, q)$. Define the first best production $q^{*}$ in the DM as the solution to $u_{x}\left(q^{*}, 0\right)+u_{h}\left(q^{*}, 0\right)=0$, and the first best real payment as $Z^{*} \equiv Z\left(q^{*}\right)$. It is straightforward to show that $Z(q)$ is strictly increasing for all $q<q^{*}$, hence $Z$ is invertible. Here, I introduce a technical condition only used occasionally to establish some stronger results:

\footnotetext{
${ }^{5}$ We nest LW as the special case with $F(H)=H$.
} 
Condition 1 Assume the solution $\arg \max _{q}\{\beta \alpha u(q, 0)-(1+\tau-\beta(1-\alpha)) Z(q)\}$ is unique.

Condition 1 guarantees that Nash bargaining solution is well-behaved such that it is never the reason to the multiplicity of equilibrium.

\section{Equilibrium}

Define an (monetary) equilibrium as follows: ${ }^{6}$

Definition 1 An equilibrium consists of prices system $\left\{w_{t}, \pi_{t+1}\right\}_{t=0}^{\infty}$, allocation $\left\{X_{t}^{i}, H_{t}^{i}, z_{t}^{i}\right\}_{t=0}^{\infty}$ for all $i \in[0,1]$ and policy $\left\{T_{t}\right\}_{t=0}^{\infty}$ such that

1. (agent optimization) given $z_{0}, w_{t}, \pi_{t+1}=\tau$ and $T_{t},\left\{X_{t}^{i}, H_{t}^{i}, z_{t+1}^{i}\right\}_{t=0}^{\infty}$ maximizes (1) and (2) subject to (3) and the bargaining solution (4)

2. (firm optimization) $H_{t}=\int_{i \in[0,1]} H_{t}^{i} d i$ maximizes (5) given $w_{t}$

3. (goods markets clear) $\int_{i \in[0,1]} X_{t}^{i} d i=F\left(H_{t}\right)$

Similarly, define a degenerate equilibrium as follows:

Definition $2 A$ degenerate equilibrium is an equilibrium such that, for any $i, j \in$ $[0,1], z_{t}^{i}=z_{t}^{j}$ for all $t$.

In a degenerate equilibrium, all agents choose the same level of money out of CM, though not necessary the same over time. Nevertheless, agents may choose different $X_{t}^{i}$ and $H_{t}^{i}$, depending on history. This formulation of degenerate equilibrium is more general than the one studied in LW which also have $X_{t}^{i}=X_{t}^{j}$ for any $i, j \in[0,1]$.

\footnotetext{
${ }^{6}$ Notice that bilateral trading in DM implies the market clearing condition is always satisfied in DM, hence is ignored in the definition of equilibrium. Also, notice that here the definition of equilibrium requires money is valuable. In general, there is always another self-fulfilling equilibrium where money is not circulating. Also, the equilibrium concept here is stronger than usual in the sense that it also requires "inflation is always a monetary phenomenon", ie, $\pi_{t}=\tau$ for all $t$. In general there can be a continuum of non-stationary equilibria.
} 


\section{The Main Result}

To ease the presentation, we first introduce a class $\mathcal{U}$ of preference and then verify the existence and uniqueness of a degenerate equilibrium for any $U \in \mathcal{U}$.

Definition $3 \mathcal{U}$ is the collection of $U$ such that for each $U \in \mathcal{U}$, for any $X \geq 0$ and $H \geq 0, U(X, H)$ satisfies

$$
U_{X X} U_{H H}-\left(U_{X H}\right)^{2}=0 \text {. }
$$

Obviously, the quasi-linear preferences studied in LW satisfy (8). Later we will solve the entire class of the exact solution to $\mathcal{U}$. Now, we are ready to summarize an important result by the following proposition

Proposition 1 Given the maintained assumptions and $\tau \geq \beta-1$. Then for any $U \in \mathcal{U}$, there always exists a degenerate equilibrium. Furthermore, if $U_{X H} \leq 0$ and condition 1 holds, then the equilibrium is unique. In particular, the degenerate equilibrium is given by $z_{i, t}^{i}=Z(q) / \theta^{*}$, where $q$ solves

$$
\frac{1+\tau-\beta}{\beta}=\alpha\left[\frac{u_{x}(q, 0)}{Z_{q}(q)}-1\right],
$$

and the Nash bargaining solution in the equilibrium is given by

$$
\begin{aligned}
& q\left(z, z^{\prime}\right)= \begin{cases}Z^{-1}\left(\theta^{*} z\right) & \text { if } z<Z^{*} / \theta^{*} \\
q^{*} & \text { if } z \geq Z^{*} / \theta^{*}\end{cases} \\
& d\left(z, z^{\prime}\right)= \begin{cases}z & \text { if } z<Z^{*} / \theta^{*} \\
Z^{*} / \Theta\left(F_{H}\left(H^{*}\right)\right) & \text { if } z \geq Z^{*} / \theta^{*}\end{cases}
\end{aligned}
$$

Proof. See Appendix.

Proposition 1 has the following interpretation. In the equilibrium, $\omega \equiv \frac{u_{x}(q, 0)}{Z_{q}(q)}-1$ is the liquidity premium (also known as bubble) of money. The equilibrium price of money is the nominal interest rate $(1+\tau) / \beta-1$. The marginal return of money is the liquidity premium $\omega$ from consumption in the DM. 
The major effort of the proof is to construct a degenerate equilibrium allocation which clears the CM goods market under heterogenous agents. The proof illustrates the seven steps to construct such a degenerate equilibrium allocation and verify its uniqueness, with the helps of three additional lemmas. Under general preferences, agents entering CM with higher money holding would choose higher $X_{t}^{i}$ and $z_{t+1}^{i}$ as well as lower $H_{t}^{i}$, and vice versa. The equilibrium allocation is constructed such that the inflation rate $\pi_{t+1}$ guarantees that each agent's budget constraint holds, the intratemporal Euler equation holds for all agents, i.e., $U_{X}\left(X_{t}^{i}, H_{t}^{i}\right)=$ $-F_{H}\left(\int_{i \in[0,1]} H_{t}^{i} d i\right) U_{H}\left(X_{t}^{i}, H_{t}^{i}\right)$, and the goods market clears, i.e., $\int_{i \in[0,1]} X_{t}^{i} d i=$ $F\left(\int_{i \in[0,1]} H_{t}^{i} d i\right)$. In LW it is straightforward since $F$ is linear and $X_{t}^{i}$ is always constant due to the assumption of quasi-linear utility: $U_{X}$ becomes independent to $H$, and both $F_{H}\left(\int_{i \in[0,1]} H_{t}^{i} d i\right)$ and $U_{H}\left(X_{t}^{i}, H_{t}^{i}\right)$ are constant. So the good market is cleared by solely $H_{t}^{i}$ which is independent to the money distribution and simply given by the agent $i$ 's budget. It is no longer the case under general preferences

Proposition 1 states that, if the money growth rate is higher than the discount rate, then (8) is a sufficient condition for the existence of monetary equilibrium, which also happens to be degenerate. The main result is consistent with the special cases under quasi-linear preferences. The sufficient condition for the degenerate equilibrium only depends on the second derivatives of $U$. Why? Consider an increase in the real money balances brought to the $\mathrm{CM}$, then, due to wealth effect, agent $i$ wants to increase $X_{t}^{i}$ and reduce $H_{t}^{i}$. Given a budget constraint, this means a tendency to decrease $z_{t+1}^{i}$. However, a wealth effect also means the agent $i$ wants to increase his consumption in the DM, hence a tendency to increase $z_{t+1}^{i}$. The two tendencies cancel each other when (8) is satisfied.

In this economy, there is an "inflation tax rate" $1+\tau-\beta$ levied on each real money balance held. With a cost $(1+\tau-\beta) Z(q)$, an agent who carries money is making an "investment" to create the expected trade surplus $\beta \alpha[u(q, 0)+u(0, q)]$ in the $\mathrm{DM}$, but some of it $\beta \alpha[Z(q)+u(0, q)]$ is extracted by the seller without bearing such cost, i.e. there is a holdup problem. So the private marginal trade surplus for the agent carrying money is given by $\beta \alpha\left[u_{x}(q, 0)-Z_{q}(q)\right]$, which is equal to the private 
marginal cost of trade with money $(1+\tau-\beta) Z_{q}(q)$. However, the social marginal trade surplus is $\beta \alpha\left[u_{x}(q, 0)+u_{h}(0, q)\right]$ and the social marginal cost of trade with money is zero. With the presence of the holdup problem, it is straightforward to verify that under $Z_{q}(q)>-u_{h}(0, q)$ for all $q \leq q^{*}{ }^{7}$ Then we have

$$
0<(1+\tau-\beta) Z_{q}(q)=\beta \alpha\left[u_{x}(q, 0)-Z_{q}(q)\right]<\beta \alpha\left[u_{x}(q, 0)+u_{h}(0, q)\right]
$$

that is the social marginal trade surplus is greater than the social marginal cost of trade with money. Thus there is under-production in this economy. In sum, the efficiency loss comes from the inflation tax and the holdup problem. Implementing the first best in the equilibrium requires inflating the economy at the discount rate and that the buyer has all the bargaining power. The holdup problem is the most severe if the seller has all the bargaining power, i.e., $\eta=0$ and $\beta \alpha[u(q, 0)-Z(q)]=0$ for all $q$. In this case, agents no longer hold any money and the monetary equilibrium does not exist.

The uniqueness of equilibrium hinges on the complementarity of consumption and leisure (negative labor), i.e., $U_{X H} \leq 0$, and the uniqueness of the solution to the holdup problem, i.e., condition 1 . If $U_{X H}>0$, ie, when consumption and leisure become substitute, then there can be a scenario where agents are indifferent between high- $X_{t}^{i}$-high- $H_{t}^{i}$ and low- $X_{t}^{i}$-low- $H_{t}^{i}$, which are both feasible even for agents with the same money holding. Hence multiple equilibria can happen. If condition 1 fails, then the holdup problem has multiple solutions, hence there can be a scenario where agents are indifferent between high- $z_{t+1}^{i}$-low- $X_{t}^{i}$ low- $H_{t}^{i}$-high- $q_{t}$ and low- $z_{t+1}^{i}$-high- $X_{t}^{i}$ high- $H_{t}^{i}$-low- $q_{t}$, so in the equilibrium agents may hold different real money balances.

\section{$5 \quad$ The Complete Solution to $\mathcal{U}$ and Examples}

In general, the whole class of exact solution to $\mathcal{U}$ is given by the following lemma:

Lemma 1 If $U \in \mathcal{U}$, then $U$ has either form:

$$
{ }^{7} \text { In particular, } Z_{q}(q) \equiv-u_{h}(0, q)+(1-\eta)\left[\frac{u_{x}(q, 0)+u_{h}(0, q)}{1-\eta\left[1+\frac{u_{x}(q, 0)}{u_{h}(0, q)}\right]}+\eta[u(q, 0)+u(0, q)] \frac{u_{x x}(q, 0) u_{h}(0, q)-u_{x}(q, 0) u_{h h}(0, q)}{u_{h}(0, q)^{2}\left[1-\eta\left[1+\frac{u_{x}(q, 0)}{u_{h}(0, q)}\right]\right]^{2}}\right] .
$$


1. $U(X, H)=\left(C_{1} X+C_{2} H+C_{3}\right) \varphi\left(\frac{C_{4} X+C_{5} H+C_{6}}{C_{1} X+C_{2} H+C_{3}}\right)+C_{7} X+C_{8} H+C_{9}$,

2. $U(X, H)=\left(C_{1} X+C_{2} H\right) \varphi\left(\frac{X}{H}\right)+C_{3} X+C_{4} H+C_{5}$,

where $C_{i}, i=1 \ldots 9$, are arbitrary constant and $\varphi$ is arbitrary $C^{m}$ function, $m \geq 2$.

Proof. See Appendix.

One might worry that $\mathcal{U}$ is still a very restrictive class of preferences. Actually several members of $\mathcal{U}$ have being commonly studied in other macro literatures, for example, it includes the class of constant return to scale (CRS) preferences. A popular member of CRS is the utility with constant elasticity of substitution (CES), which has the form $U(X, H)=\left[\alpha X^{\xi}+(1-\alpha)(1-H)^{\xi}\right]^{1 / \xi}$, for all $\xi \in(-\infty, 1)$. Lucas (1988) uses a Cobb-Douglas utility $U(X, 1-s-l)=X^{1-\sigma}(1-s-H)^{\sigma}$ to study endogenous growth. Here $A$ is the level of human capital, and $s$ the labor devoted to human capital accumulation, given by $A_{t+1}=A_{t}\left(1-\delta+a s_{t}\right)$. Heckman (1976) uses the CRS utility $U(X, A(1-s-H))$ to capture the effect of the effective leisure $A(1-s-H)$ on endogenous growth, with human capital accumulation $H_{t+1}=H_{t} g(s)$, where $g$ is some increasing and strictly concave function. Barro and Becker (1989) use the CRS utility $U(C, H)=H \widetilde{U}\left(\frac{X}{H}\right)$, where $\widetilde{U}$ is some increasing and strictly concave function, to study endogenous fertility.

The utility class $\mathcal{U}$ also includes non CRS functions. A prime example is the quasi-linear utility $U(X, H)=\widetilde{U}(X)-A H$ used in LW. Also, $\mathcal{U}$ includes the class of non-separable constant-absolute-risk-aversion (CARA) utility $U(X, H)=$ $-\exp \left(-\alpha_{X} X-\alpha_{H}(1-H)\right)$, which is widely used in, for example, asset pricing literature or economy featuring incomplete market since the associated consumption function is tractable even in a stochastic environment.

\section{Monetary Policy and Welfare}

Having established the existence and uniqueness of degenerate equilibrium, we are ready to examine the welfare consequence of monetary policy in this economy. Define 
a utilitarian welfare function as

$$
\mathcal{W}=\int_{[0,1]} \mathbb{E} \sum_{t=0}^{\infty} \beta^{t}\left\{u\left(x_{t}^{i}, h_{t}^{i}\right)+U\left(X_{t}^{i}, H_{t}^{i}\right)\right\} d i
$$

In LW, Friedman rule is optimal: a higher money growth rate always reduces welfare. Is it still true under the joint effects of general preferences and general trading protocol? The following proposition concludes that Friedman rule is still optimal in general:

Proposition 2 Given the maintained assumptions and $U \in \mathcal{U}$, fix a degenerate equilibrium, then $\delta \omega / \delta \tau \geq 0, \delta z / \delta \tau \leq 0$ and $\delta \mathcal{W} / \delta \tau \leq 0$.

\section{Proof. See Appendix}

Proposition 2 summarizes the effect of monetary policy in this economy: a higher money growth rate always increases the liquidity premium $\omega$ but reduces the equilibrium real money balance $z$. In the end, proposition 3 concludes that a higher money growth rate reduces the welfare $\mathcal{W}$. It is resulted from an important welfare feature of the utility class $\mathcal{U}$ in this economy: the welfare part from $\mathrm{CM}$ is always independent to inflation, hence the welfare can be solely ordered by the part from DM. The later is always decreasing in the inflation rate, since inflation is a tax to consumption in the DM and it worsens the holdup problem of holding money for transaction in the DM. Of course, this negative welfare consequence of money growth is not universally true. It is expected to be modified when additional frictions are introduced.

To see the intuition of why the welfare from CM is independent to inflation, notice that under the utility class $\mathcal{U}$, the fact that agents share the same marginal rate of substitution implies that they also share the same marginal utility of consumption and the same marginal disutility of labor. Hence, goods market clearing condition implies that the rise in the seller's utility (due to the wealth effect of receiving money balance from the buyer) exactly cancels the decrease in the buyer's utility, thus equilibrium money balance, hence inflation, has zero net effect on the welfare from CM.

Proposition 2 establishes a strong welfare benchmark: the optimality of Friedman rule is robust under general preferences, thought it does not necessarily implement 
the first best. The potential efficiency loss of Friedman rule comes from decentralized trading with money. The robustness of Friedman rule may or may not fail under additional frictions, which are subject to future studies.

\section{Conclusion}

In this paper, I lay out the key assumption which preserves the tractability of a microfounded monetary model under general preferences and general production. Proposition 1 establishes the unique degenerate equilibrium. Lemma 1 solves the entire class of general preferences giving rise to degenerate equilibrium. It also demonstrates how such a degenerate equilibrium can be constructed. This model also preserves many useful features of its predecessor: divisible goods, divisible money, search frictions and information frictions which give rise to money in a general equilibrium endogenously. The welfare analysis under this general preference and general trading protocol is as tractable as in LW, as demonstrated by proposition 2. The contribution of this paper is to lay out a useful environment such that a micro-founded monetary model can have broad application for later research.

Although this paper is intended to focus on Nash bargaining to have the sharpest comparison with LW, it is straight-forward to extend the main result to other bargaining solutions, for example, proportional bargaining and Kalai-Smorodinsky bargaining among many others, to the endogenous trading protocol as in $\mathrm{Hu}$ et al (2009), and even to competitive pricing in the DM. ${ }^{8}$ Also, the main result is expected to survived under other search environments, like competitive search. To save space I do not repeat the result in every single scenario. The point of this paper is to make a case that preferences is the key to establish a degenerate equilibrium.

\section{References}

[1] Aliprantis, C. D., G. Camera and D. Puzzello, 2007, "Contagion Equilibria in a Monetary Model," Econometrica

\footnotetext{
${ }^{8}$ See Kalai (1977) amd Kalai and Smorodinsky (1975) respectively.
} 
[2] Andrei, D. P. and F. Z. Valentin, 2012, "Handbook of nonlinear partial differential equations," CRC Press.

[3] Barro, R. and G. Becker, 1989, "Fertility Choice in a Model of Economic Growth," Econometrica

[4] Chiu, J., and M. Molico, 2010, "Liquidity, redistribution, and the welfare cost of inflation," Journal of Monetary Economics

[5] Faig, M., 2008, "Endogenous Buyer-Seller Choice and Divisible Money in Search Equilibrium," Journal of Economic Theory

[6] Heckman, J., 1976, "A Life-Cycle Model of Earnings, Learning, and Consumption," Journal of Political Economy

[7] Hu, T-W., J. Kennan, N. Wallace, 2009, "Coalition-Proof Trade and the Friedman Rule in the Lagos-Wright Model," Journal of Political Economy

[8] Kalai, E., 1977, "Proportional Solutions to Bargaining Situations: Interpersonal Utility Comparisons," Econometrica

[9] Kalai, E., and M. Smorodinsky, 1975, "Other solutions to Nash's bargaining problem," Econometrica

[10] Kiyotaki, N., and R. Wright, 1989, "On Money as a Medium of Exchange," Journal of Political Economy

[11] Kocherlakota, N., 1998, ""Money Is Memory," Journal of Economic Theory

[12] Lagos, R. and R. Wright, 2005, "A Unified Framework for Monetary Theory and Policy Analysis," Journal of Political Economy

[13] Lucas, R. E. Junior, 1988, "On the mechanics of economic development," Journal of Monetary Economics

[14] Meleshko, S. V., 2005, "Methods for Constructing Exact Solutions of Partial Differential Equations : Mathematical and Analytical Techniques with Applications to Engineering," Springer 
[15] Molico, M., 2006, "The Distribution Of Money And Prices In Search Equilibrium," International Economic Review

[16] Rocheteau, G., P. Rupert, K. Shell, and R. Wright, 2008, "General equilibrium with nonconvexities, sunspots, and money," Journal of Economic Theory

[17] Rocheteau, G., and R. Wright, 2005, "Money in search equilibrium, in competitive equilibrium, and in competitive search equilibrium," Econometrica

[18] Sanches, D., and S. Williamson, 2010, "Money and credit with limited commitment and theft," Journal of Economic Theory

[19] Shi, S., 1995. "Money and Prices: A Model of Search and Bargaining," Journal of Economic Theory

[20] Shi, S., 1997. "A Divisible Search Model of Fiat Money," Econometrica

[21] Trejos, A., and R. Wright, 1995, "Search, Bargaining, Money, and Prices," Journal of Political Economy

[22] Williamson, S. D., and R. Wright, 2010 "New Monetarist Economics: Models," Handbook of Monetary Economics, edited by Benjamin M. Friedman \& Michael Woodford.

\section{Appendix}

\subsection{Proof of Lemma 1}

See Andrei and Valentin (2012, P. 774). The construction of exact solution involves a method of differential constraint, which is out of the scope of this paper. A useful reference is Meleshko (2005).

\subsection{Proof of Proposition 1}

Before proving proposition, we first define homogeneous Monge-Ampere equation and establish the following lemma: 
Definition $4 U$ satisfies homogeneous Monge-Ampere equation if for any $X$ and $H$, there exists a function $\rho: \mathbb{R} \rightarrow \mathbb{R}$, where $\rho \in C^{n-1}$, such that $U$ solves either one of the following partial differential equation:

$$
\begin{gathered}
U_{H}=\rho\left(U_{X}\right), \text { or } \\
U_{X}=\rho\left(U_{H}\right) .
\end{gathered}
$$

Lemma $2 U \in \mathcal{U}$ if and only if $U$ satisfies homogeneous Monge-Ampere equation. If $U_{X X}<0$, then $U \in \mathcal{U}$ satisfies (10).

Proof. To prove the "if" part of the lemma, fix any $U$ satisfying homogeneous MongeAmpere equation. Consider the case of (10). Then differentiate both sides of (10) with respect to $X$ and to $H$, we have:

$$
\begin{aligned}
& U_{X H}=\rho^{\prime}\left(U_{X}\right) U_{X X}, \\
& U_{H H}=\rho^{\prime}\left(U_{X}\right) U_{X H},
\end{aligned}
$$

where $\rho^{\prime}$ exists as $\rho \in C^{n-1}$. Suppose $\rho^{\prime} \neq 0$, then multiplying the left hand side of the first equation to the right hand side of the second equation, we have $U_{X X} U_{H H}-$ $\left(U_{X H}\right)^{2}=0$, hence $U$ satisfies Monge-Ampere equation. Suppose $\rho^{\prime}=0$, then the above implies $U_{X H}=U_{H H}=0$, hence again we have $U_{X X} U_{H H}-\left(U_{X H}\right)^{2}=0$. The same proof for the case of (11).

If $U_{X X} \neq 0$ except on a set of zero measure, then $U_{H X} / U_{X X}$ is well-defined almost surely. Fix some $\varepsilon>0$. For any $U_{X}(X, H)$, define $x(t)$ and $h(t)$ as function in $t$ such that $x(0)=h(0)=\varepsilon, x(1)=X$ and $h(1)=H$. Then we can construct $\rho\left(U_{X}(X, H)\right)$ as a path integral of $t$ over $[0,1]$ :

$$
\begin{aligned}
\rho\left(U_{X}(X, H)\right) & \equiv \int_{0}^{1} \frac{U_{H X}(x(t), h(t))}{U_{X X}(x(t), h(t))} d U_{X}(x(t), h(t))+U_{H}(\varepsilon, \varepsilon), \\
& =\int_{0}^{1} U_{H X}(x(t), h(t)) d x(t)+\int_{0}^{1} \frac{U_{H X}(x(t), h(t))^{2}}{U_{X X}(x(t), h(t))} d h(t)+U_{H}(\varepsilon, \varepsilon), \\
& =\int_{0}^{1} U_{H X}(x(t), h(t)) d x(t)+\int_{0}^{1} U_{H H}(x(t), h(t)) d h(t)+U_{H}(\varepsilon, \varepsilon), \\
& =U_{H}(X, H),
\end{aligned}
$$


where the second line utilizes the fact that $U_{X X} \neq 0$ and $U_{H H}=\left(U_{X H}\right)^{2} / U_{X X}$ for any $U \in \mathcal{U}$. Thus $U$ always satisfies (10). Similar construction for the case of $U_{H H} \neq 0$ and then we have $U$ always satisfying (11). Finally, if $U_{X X}=0$ or $U_{H H}=0$ on a set of positive measure, then we have constant $U_{X}$ or constant $U_{H}$, hence $\rho$ is a constant function, so either (10) or (11) is satisfied.

Finally, it is straightforward to verify the "only if" part that $U$ of the form in Lemma 1 implies $U$ satisfying homogeneous Monge-Ampere equation.

Lemma 3 Given the maintained assumptions and $U \in \mathcal{U}$. Suppose an equilibrium exists. Fix $w_{t}$, then there exists positive function $\Theta(w)$ such that for all $z$

$$
U_{X}\left(X_{t}^{i}, H_{t}^{i}\right)=\Theta\left(w_{t}\right)
$$

Also, the value function have the form

$$
V_{t}(z)=\Theta\left(w_{t}\right) z+\text { constant }
$$

Furthermore, if $U_{X H} \leq 0$, then such $\Theta(w)$ exists and is unique even off-equilibrium path.

Proof. Given lemma 2, the first order conditions w.r.t. $X$ and $H$ of the Bellman equation (2) imply

$$
w_{t}=\frac{-\rho\left(U_{X}\left(X_{t}^{i}, H_{t}^{i}\right)\right)}{U_{X}\left(X_{t}^{i}, H_{t}^{i}\right)} .
$$

Since the right hand side only depends on $U_{X}\left(X_{t}^{i}, H_{t}^{i}\right)$, we have (13) given an equilibrium exists.

To show (14), apply an envelope theorem then we have $V_{z, t}(z)=U_{X}\left(X_{t}^{i}, H_{t}^{i}\right)=$ $\varphi\left(w_{t}\right)$. Then integrating $V_{z, t}(z)$ over $z$ we have (14).

To show uniqueness of $\Theta\left(w_{t}\right)$, since $U_{H}<0$, we have $\rho(\theta)<0$. Since $U_{X X} \leq 0$ and $U_{X H} \leq 0$, then from (12) we have $\rho^{\prime}(\theta) \geq 0$, then the right hand side of the above is strictly decreasing in $U_{X}\left(X_{t}^{i}, H_{t}^{i}\right)$. Since $U$ satisfies Inada condition, the right hand side ranges from zero to infinity. Thus, there exists unique positive function $\Theta(w)$ that solves $U_{X}\left(X_{t}^{i}, H_{t}^{i}\right)=\Theta\left(w_{t}\right)$. 
Then we are ready to solve the Nash bargaining solution (4), which is given by the following lemma:

Lemma 4 Given the maintained assumptions and $U \in \mathcal{U}$. Suppose an equilibrium exists. Then the Nash bargaining solution $\left(q_{t}\left(z, z^{\prime}\right), d_{t}\left(z, z^{\prime}\right)\right)$ to $(4)$ is given by

$$
\begin{aligned}
& q_{t}\left(z, z^{\prime}\right)= \begin{cases}Z^{-1}\left(\Theta\left(w_{t}\right) z\right) & \text { if } z<Z^{*} / \Theta\left(w_{t}\right) \\
q^{*} & \text { if } z \geq Z^{*} / \Theta\left(w_{t}\right)\end{cases} \\
& d_{t}\left(z, z^{\prime}\right)= \begin{cases}z & \text { if } z<Z^{*} / \Theta\left(w_{t}\right) \\
Z^{*} / \Theta\left(w_{t}\right) & \text { if } z \geq Z^{*} / \Theta\left(w_{t}\right)\end{cases}
\end{aligned}
$$

Proof. Substituting (14) into (4), then the first order condition w.r.t. $q_{t}$ is given by

$$
\Theta\left(w_{t}\right) z=-u\left(0, q_{t}\right)+(1-\eta) \frac{u\left(q_{t}, 0\right)+u\left(0, q_{t}\right)}{1-\eta\left[1+\frac{u_{x}\left(q_{t}, 0\right)}{u_{h}\left(0, q_{t}\right)}\right]}, \text { if } z<Z^{*} / \Theta\left(w_{t}\right)
$$

otherwise if $z \geq Z^{*} / \Theta\left(w_{t}\right)$, then we have $q_{t}=q^{*}$ and $d_{t}=Z^{*} / \Theta\left(w_{t}\right)$.

Proof of proposition 1. Now, we are ready to sketch the proof of proposition 1. We construct a degenerate equilibrium and then verify its uniqueness. To construct a degenerate equilibrium, due to the concavity of the problem, we only need to verify that there exists allocations $\left\{X_{t}^{i}, H_{t}^{i}, z_{t}\right\}_{t=0}^{\infty}$ satisfying transversality condition (omitted here as it can be easily checked) as well as solving all first order conditions, agent $i$ 's budget constraint and goods market clearing condition under some prices system $\left(w_{t}, \pi_{t+1}\right)$. Hence we need to show that there exists $\left\{X_{t}^{i}, H_{t}^{i}, z_{t}\right\}_{t=0}^{\infty}$ satisfying all the following first order conditions of $X_{t}^{i}, H_{t}^{i}$ and $z_{t+1}^{i}$, and goods market clearing condition:

$$
\begin{gathered}
w_{t} U_{X}\left(X_{t}^{i}, H_{t}^{i}\right)=-U_{H}\left(X_{t}^{i}, H_{t}^{i}\right), \\
w_{t}=F_{H}\left(H_{t}\right), \text { where } H_{t}=\int_{i \in[0,1]} H_{t}^{i} d i \\
\frac{1+\tau}{\beta} \frac{\Theta\left(w_{t-1}\right)}{\Theta\left(w_{t}\right)}=\left\{\begin{array}{l}
\alpha \frac{u_{x}\left(q_{t}, 0\right)}{Z_{q}\left(q_{t}\right)}+1-\alpha \text { if } z_{t}<Z^{*} / \Theta\left(w_{t}\right) \\
1-\alpha \text { if } z_{t} \geq Z^{*} / \Theta\left(w_{t}\right)
\end{array}\right. \\
\int_{i \in[0,1]} X_{t}^{i} d i=F\left(H_{t}\right)
\end{gathered}
$$


as well as agents budget (3) after substituting (5), (6) and (17):

$$
X_{t}^{i}=\left\{\begin{array}{l}
F_{H}\left(H_{t}\right)\left(H_{t}^{i}-H_{t}\right)+F\left(H_{t}\right)-z_{t}, \text { for a buyer } \\
F_{H}\left(H_{t}\right)\left(H_{t}^{i}-H_{t}\right)+F\left(H_{t}\right)+z_{t}, \text { for a seller } \\
F_{H}\left(H_{t}\right)\left(H_{t}^{i}-H_{t}\right)+F\left(H_{t}\right), \text { for a no-matcher }
\end{array}\right.
$$

Notice that (18) has made use of (14) from lemma 3.

First, by assumption 2 there is unique $X^{*}$ and $H^{*}$ such that $F_{H}\left(H^{*}\right) U_{X}\left(X^{*}, H^{*}\right)=$ $-U_{H}\left(X^{*}, H^{*}\right)$. Set $\theta^{*} \equiv U_{X}\left(X^{*}, H^{*}\right), H_{t}=H^{*}$ and $w_{t}=F_{H}\left(H^{*}\right)$. Thus the firm's first order condition (17) is satisfied.

Second, set $U_{X}\left(X_{t}^{i}, H_{t}^{i}\right)=\theta^{*}$. Notice that there exists some $q$ satisfying (21):

$$
\frac{1+\tau-\beta}{\beta}=\alpha\left[\frac{u_{x}(q, 0)}{Z_{q}(q)}-1\right] \text { and } Z(q)<Z^{*}
$$

To see the existence of such $q$, consider the two limit cases: $q=q^{*}$ and $q=0$. Evaluate $q=q^{*}$, then we have

$$
\frac{Z_{q}\left(q^{*}\right)}{u_{x}\left(q^{*}, 0\right)} \equiv 1+(1-\eta) \eta\left[u\left(q^{*}, 0\right)+u\left(0, q^{*}\right)\right] \frac{u_{x x}\left(q^{*}, 0\right)+u_{h h}\left(0, q^{*}\right)}{-u_{h}\left(0, q^{*}\right)^{2}}>1
$$

so the right hand side of $(21)$ at $q=q^{*}$ is negative. Evaluate $q=0$, then we have

$$
\lim _{q \rightarrow 0} Z_{q}(0)=\frac{(1-\eta) \lim _{q \rightarrow 0} u_{x}(q, 0)}{1-\eta\left[1+\lim _{q \rightarrow 0} \frac{u_{x}(q, 0)}{u_{h}(0, q)}\right]}
$$

So the right hand side of (21) at $q=0$ is infinite. Since $\tau \geq \beta-1$ and hence the left hand side of (21) is finite and positive, the fact that the right hand side of (21) is continuous in $q$ implies there exists some $q$ satisfying (21).

Set $z_{t}=Z(q) / \theta^{*}$. Then given lemma 2, the first order condition (18) becomes (21), which is automatically satisfied by construction of $z$.

Third, it is straight-forward to verify from lemma 1 that for any $U \in \mathcal{U}, U_{X}$ always has the form

$U_{X}(X, H)=C_{1} \varphi\left(\frac{C_{4} X+C_{5} H+C_{6}}{C_{1} X+C_{2} H+C_{3}}\right)+\left(C_{4}+C_{1} \frac{C_{4} X+C_{5} H+C_{6}}{C_{1} X+C_{2} H+C_{3}}\right) \varphi^{\prime}\left(\frac{C_{4} X+C_{5} H+C_{6}}{C_{1} X+C_{2} H+C_{3}}\right)+C_{7}$ 
if $U$ is of the form in lemma 1.1, or

$$
U_{X}(X, H)=C_{1} \varphi\left(\frac{X}{H}\right)+\left(C_{1} \frac{X}{H}+C_{2}\right) \varphi^{\prime}\left(\frac{X}{H}\right)+C_{3},
$$

if $U$ is of the form in lemma 1.2. In either case, $U_{X}$ only depends on a ratio of two linear functions of $X$ and $H$. Thus, fix some $\theta_{i, t}$, for any $X_{t}^{i}$ and $H_{t}^{i}$ satisfying $\theta_{i, t}=U_{X}\left(X_{t}^{i}, H_{t}^{i}\right)$, there is a function $\gamma(\theta)$ such that

$$
\gamma\left(\theta_{i, t}\right)=\frac{C_{4} X_{t}^{i}+C_{5} H_{t}^{i}+C_{6}}{C_{1} X_{t}^{i}+C_{2} H_{t}^{i}+C_{3}},
$$

if $U$ is of the form in lemma 1.1, or

$$
\gamma\left(\theta_{i, t}\right)=\frac{X_{t}^{i}}{H_{t}^{i}}
$$

if $U$ is of the form in lemma 1.2. It implies a linear relationship between $X_{t}^{i}$ and $H_{t}^{i}$

$$
X_{t}^{i}=D_{1}\left(\theta_{i, t}\right) H_{t}^{i}+D_{2}\left(\theta_{i, t}\right)
$$

where $D_{1}(\theta)$ and $D_{2}(\theta)$ are some linear function depending on $\gamma(\theta)$ only. Notice $D_{1}\left(\theta_{i, t}\right) \leq 0$ if $U_{X H} \leq 0$, since so to keep $\theta_{i, t}=U_{X}\left(X_{t}^{i}, H_{t}^{i}\right)$, any increase in $H_{t}^{i}$ must not lead to increase in $X_{t}^{i}$.

Forth, set $\left(X_{t}^{i}, H_{t}^{i}, z_{t+1}^{i}\right)=\left(X^{b}, H^{b}, z\right)$ for a buyer in the DM of period $t,\left(X_{t}^{i}, H_{t}^{i}, z_{t+1}^{i}\right)=$ $\left(X^{s}, H^{s}, z\right)$ for a seller in the DM of period $t$ and $\left(X_{t}^{i}, H_{t}^{i}, z_{t+1}^{i}\right)=\left(X^{*}, H^{*}, z\right)$ for a no-matcher in the DM of period $t$, which are given by

$$
\begin{aligned}
H^{b} & =H^{*}-\frac{z}{D_{1}\left(\theta^{*}\right)-F_{H}\left(H^{*}\right)}, \\
H^{s} & =H^{*}+\frac{z}{D_{1}\left(\theta^{*}\right)-F_{H}\left(H^{*}\right)}, \\
X^{b} & =D_{1}\left(\theta^{*}\right) H^{b}+D_{2}\left(\theta^{*}\right), \\
X^{s} & =D_{1}\left(\theta^{*}\right) H^{s}+D_{2}\left(\theta^{*}\right),
\end{aligned}
$$

Assumption 2 guarantees the above is well-defined. To see, suppose not, then it must be that the buyer cannot afford to hold real money balances $z$, i.e., for any $(X, H)$ 
such that $\theta^{*}=U_{X}(X, H)$, we have

$$
z>X^{*}-X+F_{H}\left(H^{*}\right)\left(H-H^{*}\right)
$$

Notice that the Nash bargaining solution implies $u\left(q^{*}, 0\right)-\theta^{*} z>0$, then combining this and (29), we have

$$
\frac{u\left(q^{*}, 0\right)}{\theta^{*}}>z \geq \max _{X, H}\left\{X^{*}-X+F_{H}\left(H^{*}\right)\left(H-H^{*}\right)\right\} \text { s.t. } \theta^{*}=U_{X}(X, H),
$$

which contradicts to assumption 2 .

Then notice that since $U \in \mathcal{U}$, assumption 3 and (24) imply

$$
X^{*}=D_{1}\left(\theta^{*}\right) H^{*}+D_{2}\left(\theta^{*}\right)
$$

Then using (25)-(30) to substitute out $D_{1}\left(\theta^{*}\right)$ and $D_{2}\left(\theta^{*}\right)$, it is straight-forward to verify agents' budget (20) is satisfied.

Fifth, notice that here we construct $X^{b}$ and $X^{s}$ such that (24) is satisfied with $\theta^{*}=U_{X}\left(X_{t}^{i}, H_{t}^{i}\right)$. By lemma 2 we have $U_{H}\left(X_{t}^{i}, H_{t}^{i}\right)=\rho\left(U_{X}\left(X_{t}^{i}, H_{t}^{i}\right)\right)$. Also, notice that assumption 3 implies $w_{t} \theta^{*}=-\rho\left(\theta^{*}\right)$. Thus combining the above we have the first order condition (16) satisfied.

Sixth, since there are measure $\alpha$ of buyers, measure $\alpha$ of sellers, and measure $1-2 \alpha$ of no-matchers, summing (27)-(30) times the corresponding measures we have $\int_{i \in[0,1]} X_{t}^{i} d i=X^{*}$, hence by assumption 3 , the goods market clearing condition (19) is satisfied. Since all first order conditions and market clearing condition are satisfied, thus the allocation constructed above is indeed a degenerate equilibrium.

Finally, to show the uniqueness of equilibrium, notice that (16), (17), (19), (20) are still valid first order conditions and market clearing conditions for any equilibrium. Given $U_{X H} \leq 0$, then by lemma 3 , we always have $U_{X}\left(X_{t}^{i}, H_{t}^{i}\right)=U_{X}\left(X_{t}^{i}, H_{t}^{i}\right)=\theta_{t}$ for any $i, j \in[0,1]$. Hence we can replace (16) with (24). Then summing (24) for all $i \in[0,1]$ with $\theta_{i, t}=\theta_{t}$, in an equilibrium we must have

$$
X_{t}=\int_{[0,1]} X_{t}^{i} d i=D_{1}\left(\theta_{t}\right) H_{t}+D_{2}\left(\theta_{t}\right)=F\left(H_{t}\right)
$$


Then by assumption 2, we must have $H_{t}=H^{*}$ and $\theta_{t}=\theta^{*}$ as the unique solution to keep all the above held with equality. In other words, satisfying (24) and (19) imply $H_{t}=H^{*}$ and $\theta_{t}=\theta^{*}$, where (17) is automatically satisfied. Since $V_{z, t}(z)=$ $U_{X}\left(X_{t}^{i}, H_{t}^{i}\right)=\theta_{t}=\theta^{*}$ for all $i$ and $z$, then (18) is replaced by a general form:

$$
\max _{q}\{-(1+\tau-\beta(1-\alpha)) Z(q)+\beta \alpha u(q, 0)\}
$$

which under condition 1 there is unique solution $q$, hence unique real money balances $z=Z(q) / \theta^{*}$. Finally since the agents' budget (20) and (24) are both linear $X_{t}^{i}$ and $H_{t}^{i}$, then either the solution does not exist, or (20) and (24) are the same and any $X_{t}^{i}$ and $H_{t}^{i}$ go, or they jointly solve unique $X_{t}^{i}$ and $H_{t}^{i}$. The first case is ruled out as we have established the existence of an equilibrium. The second case is also ruled out as (20) implies $X_{t}^{i}$ is increasing in $H_{t}^{i}$ but (24) implies $X_{t}^{i}$ is non-increasing in $H_{t}^{i}$ due to $U_{X X}<0$ and $U_{X H} \leq 0$. Thus the equilibrium allocation is unique.

\subsection{Proof of Proposition 2}

Suppose $U$ is of the form in lemma 1.1. Recall from the proof of proposition 1 that (9) is the first order condition to (31). The second order necessary condition implies

$-(1+\tau-\beta(1-\alpha)) Z_{q q}(q)+\beta \alpha u_{x x}(q, 0) \leq 0 \Rightarrow \frac{u_{x x}(q, 0) Z_{q}(q)-u_{x}(q, 0) Z_{q q}(q)}{Z_{q}(q)^{2}} \leq 0$.

Hence, by totally differentiating (9), we have $d q / d \tau \leq 0$ and $\partial \omega / \partial \tau \geq 0$. Since $z=Z(q) / \theta^{*}$, then we have $d z / d \tau \leq 0$. Also $U$ of the form in lemma 1.1 implies the utility in the degenerate equilibrium becomes

$$
U\left(X_{t}^{i}, H_{t}^{i}\right)=\left(C_{1} X_{t}^{i}+C_{2} H_{t}^{i}+C_{3}\right) \varphi\left(\gamma\left(\theta^{*}\right)\right)+C_{7} X_{t}^{i}+C_{8} H_{t}^{i}+C_{9}
$$

where $\gamma$ is given by (22). Summing the utility over agents, we have

$$
\int_{[0,1]} U\left(X_{t}^{i}, H_{t}^{i}\right) d i=\left(C_{1} F\left(H^{*}\right)+C_{2} H^{*}+C_{3}\right) \varphi\left(\gamma\left(\theta^{*}\right)\right)+C_{7} F\left(H^{*}\right)+C_{8} H^{*}+C_{9}
$$


which is independent to $\tau$. Hence, the welfare in the degenerate equilibrium becomes

$$
\mathcal{W}=\frac{\alpha}{1-\beta}[u(q, 0)+u(0, q)]+\text { constant } .
$$

Thus the comparative statics on $\mathcal{W}$ of $z$ is

$$
\frac{\partial \mathcal{W}}{\partial q}=\frac{\alpha}{1-\beta}\left[\frac{u_{x}(q, 0)+u_{h}(0, q)}{Z_{q}(q)}\right] \geq 0,
$$

hence given $d q / d \tau \leq 0$ we have $\partial \mathcal{W} / \partial \tau=(\partial \mathcal{W} / \partial q)(d q / d \tau) \leq 0$. 